\title{
The influence of pore size and stiffness on tenocyte bioactivity and transcriptomic stability in collagen-GAG scaffolds
}

\author{
William K. Grier ${ }^{1}$, Ehiremen M. Iyoha ${ }^{1}$, Brendan A.C. Harley ${ }^{1,2^{*}}$ \\ ${ }^{1}$ Dept. of Chemical \& Biomolecular Engineering \\ ${ }^{2}$ Carl R. Woese Institute for Genomic Biology \\ University of Illinois at Urbana-Champaign \\ Urbana, IL 61801
}

\author{
Corresponding Author: \\ B.A.C. Harley \\ Dept. of Chemical and Biomolecular Engineering \\ Carl R. Woese Institute for Genomic Biology \\ University of Illinois at Urbana-Champaign \\ 110 Roger Adams Laboratory \\ 600 S. Mathews Ave. \\ Urbana, IL 61801 \\ Phone: (217) 244-7112 \\ Fax: (217) 333-5052 \\ email: bharley@illinois.edu
}

\section{Author contributions:}

W.K.G. and B.A.C.H designed the research; W.K.G. and E.M.I. performed the research; W.K.G., E.M.I., and B.A.C.H. analyzed the data; W.K.G. and B.A.C.H. wrote the manuscript.

Funding. This work was supported by the National Institutes of Health (R03 AR062811 and R21 AR063331 to BACH; T32GM070421 to WKG) and the National Science Foundation (REU to EMI under CBET-0939511). 


\begin{abstract}
Orthopedic injuries, particularly those involving tendons and ligaments, are some of the most commonly treated musculoskeletal ailments, but are associated with high costs and poor outcomes. A significant barrier in the design of biomaterials for tendon tissue engineering is the rapid de-differentiation observed for primary tenocytes once removed from the tendon body. Herein, we evaluate the use of an anisotropic collagen-glycosaminoglycan (CG) scaffold as a tendon regeneration platform. We report the effects of structural properties of the scaffold (pore size, collagen fiber crosslinking density) on resultant tenocyte bioactivity, viability, and gene expression. In doing so we address a standing hypothesis that scaffold anisotropy and strut flexural rigidity (stiffness) co-regulate long-term maintenance of a tenocyte phenotype. We report changes in equine tenocyte specific gene expression profiles and bioactivity across a homologous series of anisotropic collagen scaffolds with defined changes in pore size and crosslinking density. Anisotropic scaffolds with higher crosslinking densities and smaller pore sizes were more able to resist cell-mediated contraction forces, promote increased tenocyte metabolic activity, and maintain and increase expression of a tenogenic gene expression profiles. These results suggest that control over scaffold strut flexural rigidity via crosslinking and porosity provides an ideal framework to resolve structure-function maps relating the influence of scaffold anisotropy, stiffness, and nutrient biotransport on tenocyte-mediated scaffold remodeling and long-term phenotype maintenance.
\end{abstract}

Key words: collagen; scaffold; pore size; crosslinking; tenocyte; bioactivity 


\section{Introduction}

Geometrically and mechanically anisotropic tissues present unique challenges for the field of tissue engineering. The functional capacity of tendon derives from its extracellular matrix (ECM) composed of type I collagen arranged in highly aligned cross-linked fibrils (James et al., 2008; Liu et al., 2008; Towler and Gelberman, 2006). The cellular component of tendon is primarily composed of tendon fibroblasts, or tenocytes, distributed throughout a hierarchical organization of aligned type I collagen fibers (James et al., 2008; Liu et al., 2008). Tendon and ligament injuries plague individuals from all walks of life, from elite athletes to the elderly, with more than 32 million occurrences every year in the US alone (Breidenbach et al., 2013; James et al., 2008; Liu et al., 2008; Xu and Murrell, 2008). While small tendon injuries heal spontaneously via regeneration, larger defects undergo a repair-mediated process generating fibrocartilagenous scar tissue with inferior structural and biomechanical properties. The resulting misaligned ECM leads to losses in range of motion and strength along with high rates of injury recurrence (James et al., 2008; Liu et al., 2008; Xu and Murrell, 2008). Inadequate healing after surgical repair remains a primary clinical challenge. Because the musculoskeletal system depends on a balance of contributions from multiple tissues to maintain joint patency, ineffective tendon repair has both short-term (i.e. slowed rehabilitation) and long-term (i.e. chronic, degenerative joint pathologies) consequences (Butler et al., 2008; Liu et al., 2008). Due to an ageing US population with increasing rates of underlying chronic conditions (i.e. obesity, diabetes), injury volume and complexity are only expected to increase (Fox et al., 2011).

A primary challenge to improving tendon regeneration is poor understanding of how tenocytes respond to the dynamic structural microenvironment within tendon. Tendon fibroblasts, or 
tenocytes (TCs) are responsible for tendon homeostasis, remodeling, and repair. However, tenocytes exhibit rapid de-differentiation when cultured in 2D or within hydrogels (Clegg et al., 2007; Taylor et al., 2009). While tensile loading and aligned topographical cues on twodimensional substrates can partially abrogate this effect (Caporali et al., 2009; Maeda et al., 2009; Paxton et al., 2011), 3D platforms for stable expansion of tenocytes represent an important advance for the field. A primary effort in the development of tissue engineering and regenerative medicine approaches for a range of tissues is therefore the design of an appropriate biomaterial platform. Often these approaches are inspired by the native extracellular matrix (ECM) in order to provide an environment to speed healing or regeneration (Spilker et al., 2001; Yannas, 2001; Yannas et al., 1989).

Collagen-glycosaminoglycan (CG) scaffolds have been used in a wide variety of in vivo applications for skin, peripheral nerve, and cartilage tissue engineering as well as 3D environments for in vitro studies of cell-matrix interactions (cell, migration and contraction) behavior (Farrell et al., 2006; Harley et al., 2007a; Harley et al., 2008; Harley et al., 2004; O'Brien et al., 2005; Schulz Torres et al., 2000; Yannas et al., 1989). The vast majority of past efforts using CG scaffolds have focused primarily on the regeneration of soft tissues. However, those scaffold variants were unsuitable for tendon repair applications due to their inability to withstand TC-mediated contraction (Caliari and Harley, 2011; Schulz Torres et al., 2000) leading to rapid tenocyte de-differentiation. We have recently developed a fabrication method to produce anisotropic CG scaffolds composed of an aligned ellipsoidal pore structure (Caliari and Harley, 2011). This anisotropic scaffold geometry promotes preferential tenocyte alignment along the long axis of the ellipsoidal pores. Interestingly, increasing the scaffold relative density while 
maintaining the aligned pore geometry was shown to reduce TC-mediated scaffold contraction, maintain an aligned morphology, and reduce TC de-differentiation (Caliari and Harley, 2011; Caliari et al., 2011; Caliari et al., 2012). The potential to tailor scaffold anisotropy and its resistance to adverse (contraction-based) remodeling events offers an exciting platform to control long-term maintenance of tenocyte bioactivity. Here, we look at two different means to alter the local scaffold environment experienced by individual tenocytes: pore size and crosslinking density. For a low-density open-cell foam such as the CG scaffold, changing scaffold pore size does not affect macro-scale mechanical properties such as scaffold elastic modulus (Gibson et al., 2010; Harley et al., 2007c); however, changing the pore size for a series of scaffolds with constant relative density does alter the length and thickness (hence flexural rigidity) of individual struts which define the pore structure of the scaffold (Harley et al., 2007a). Alternatively, increasingly scaffold crosslinking density via either dehydrothermal (DHT) (Gibson et al., 2010; Harley et al., 2007c; Olde Damink et al., 1996; Schulz Torres et al., 2000) or chemical (1-ethyl3-[3-dimethylaminopropyl] carbodiimide hydrochloride, EDC; N-hydroxysulfosuccinimide, NHS) (Olde Damink et al., 1996) means can increase scaffold and individual strut stiffness independent of pore size. Both modifications alter the local strut properties but via different means, opening the door to questions exploring how strut stiffness versus pore size affects TCmediated contraction and resultant maintenance of TC-phenotype.

The work presented here seeks to describe the relationship between scaffold pore size and crosslinking with scaffold mechanical properties and its ability to resist TC-mediated contraction. We hypothesized that scaffold's ability to resist TC-mediated contraction would increase with increasing crosslinking density and decreasing pore size. Moreover, more 
mechanically robust scaffolds with smaller pore sizes and higher crosslinking densities were hypothesized to be more supportive of the maintenance of a tendon-like phenotype through the resistance to TC-mediated contraction and preservation of a permeable open-cell pore structure that allows for efficient nutrient transport and cell spreading. While our group and others have investigated the impact of initial scaffold pore size and crosslinking on cell attachment and tenocyte contraction (Caliari and Harley, 2011; Caliari et al., 2012; Schulz Torres et al., 2000), this previous work only considered cell-mediated contraction (but not genomic stability) as an endpoint (Schulz Torres et al., 2000) or did not fully examine the combinatorial effect of EDC crosslinking density and scaffold pore size on TC-mediated contraction and subsequent bioactivity (Caliari and Harley, 2011; Caliari et al., 2012). As a result, in this manuscript we explored the combined effect of scaffold crosslinking density and pore size on tenocyte-mediated contraction and genomic stability, using strut flexural rigidity as a means to consider the results.

\section{Materials \& Methods}

\section{Preparation of CG suspension}

Type I microfibrillar collagen from bovine tendon (Sigma-Aldrich, St. Louis, MO) and chondroitin-4,6-sulfate from shark cartilage (Sigma-Aldrich, St. Louis, MO) were homogenized in $0.05 \mathrm{M}$ acetic acid. The concentration of the collagen suspension made was $1.0 \%$, and the ratio of the collagen to glycosaminoglycan was kept at 11.25:1 (Caliari and Harley, 2011; O’Brien et al., 2004; Yannas et al., 1989).

\section{Aligned CG scaffold fabrication}


The scaffolds were fabricated through directional solidification as previously described (Caliari and Harley, 2011). Degassed CG suspension was pipetted into a polytetrafluoroethylene (PTFE)copper mold (8mm diameter, 15mm deep), and placed on a pre-cooled freeze-dryer shelf (VirTis, Gardner, NY). The mismatch in thermal conductivity between the PTFE body and copper base of the mold promotes unidirectional heat transfer through the more conductive copper, resulting in anisotropic ice crystal formation when cooled (Caliari and Harley, 2011). In order to control pore size, the CG suspension was frozen at three different temperatures $\left(\mathrm{T}_{\mathrm{f}}\right):-10^{\circ} \mathrm{C},-40^{\circ} \mathrm{C}$, and $-60^{\circ} \mathrm{C}$. These freezing temperatures have been previously shown to produce scaffolds with aligned pore geometries of 243, 152, and $55 \mu \mathrm{m}$ diameters respectively (Caliari and Harley, 2011). The CG suspensions were frozen for $2 \mathrm{~h}$, and then sublimated at $0^{\circ} \mathrm{C}$ and $200 \mathrm{mTorr}$ to remove the ice crystals, resulting in a dry porous scaffold.

\section{Aligned CG scaffold crosslinking}

Following lyophilization, the scaffolds were DHT crosslinked in a vacuum oven (Welch, Niles, IL) $\left(105^{\circ} \mathrm{C},<25\right.$ torr $)$ for $24 \mathrm{~h}$ and then stored in a desiccator until use. Before use, scaffolds were immersed in 200-proof ethanol for 6 hours, followed by washing with phosphate-buffered saline (PBS) overnight. Solutions of 1-ethyl-3-[3-dimethylaminopropyl] carbodiimide hydrochloride (Sigma-Aldrich, St. Louis, MO) and N-hydroxysulfosuccinimide (Sigma-Aldrich) were used to further crosslink each of the three scaffolds groups at three different molar ratios: 1:1:5 (low), 5:2:5 (medium), and 5:2:1 (high) EDC:NHS:COOH that correspond with increasing collagen strut and overall scaffold stiffness (Harley et al., 2007c; Olde Damink et al., 1996). While characterization of the pore size (Caliari and Harley, 2011) and relative changes in modulus (E5:2:1 3.6-fold greater than E1:1:5) (Harley et al., 2007b) and swelling ratio (EDC1:1:5 
1.6-fold greater swelling than EDC5:2:5) (Vickers et al., 2010) with crosslinking have been previously published, a summary of properties for the library of scaffolds tested here is provided in Table 1.

\section{Equine tenocyte isolation and culture}

Equine tenocytes were isolated as previously described (Caliari and Harley, 2011) from horses aged 2-3 years euthanized for reasons not related to tendinopathy and in a manner consistent with protocols approved by the University of Illinois IACUC. Digital flexor tendons were extracted, diced, and incubated in trypsin-ethylenediaminetetraacetic acid (Invitrogen, Carlsbad, CA) then $0.15 \%$ collagenase II (Worthington, Lakewood, NJ) overnight at $37^{\circ} \mathrm{C}$ under constant shaking. Digest solution was filtered (40 $\mu \mathrm{m}$ pore size) to isolate tenocytes (Kapoor et al., 2010). All cell concentrations were determined using a Bright-Line hemocytometer (Sigma Aldrich). Tenocytes were plated at a density of $1 \times 10^{4}$ cells $/ \mathrm{cm}^{2}$, and cultured in high glucose Dulbecco's modified Eagle’s medium (DMEM, Fisher, Pittsburgh, PA) with 1\% L-glutamine (Invitrogen, Carlsbad, CA) supplemented with 10\% fetal bovine serum (FBS, Invitrogen, Carlsbad, CA), 1\% penicillin/streptomycin (Invitrogen, Carlsbad, CA), 1\% amphotericin-B (MP Biomedical, Solon, $\mathrm{OH}$ ), and $25 \mu \mathrm{g} / \mathrm{mL}$ ascorbic acid (Wako, Richmond, VA) (Caliari and Harley, 2011). The tenocytes were cultured at $37^{\circ} \mathrm{C}$ and $5 \% \mathrm{CO}_{2}$, and were fed every 3 days. Cells were used at passage 3.

\section{Scaffold seeding}

Each cylindrical CG scaffold (8mm diameter, 15mm length) was cut into 5mm long plugs (8mm dia.), and placed in ultra-low attachment 6-well plates (Corning Life Sciences, Lowell, 
MA). Confluent tenocytes were trypsinized and resuspended at a concentration of $5 \times 10^{5}$ cells $/ 20 \mu \mathrm{L}$ media. Scaffolds were seeded with tenocytes using a previously validated static seeding method (Caliari and Harley, 2011; O'Brien et al., 2005) with $10 \mu \mathrm{L}$ of cell suspension $\left(2.5 \times 10^{5}\right.$ cells) pipetted directly onto each scaffold. The scaffolds were then incubated at $37^{\circ} \mathrm{C}$ for 15 minutes, turned over, and seeded with an additional $10 \mu \mathrm{L}$ of cell suspension for a total of $5 \times 10^{5}$ cells seeded per scaffold. The cell-seeded scaffolds were then incubated at $37^{\circ} \mathrm{C}$ with $5 \%$ $\mathrm{CO}_{2}$ and fed complete DMEM every 3 days (Caliari and Harley, 2011).

\section{Quantification of cell metabolic activity}

The mitochondrial metabolic activity of the tenocytes within each scaffold was determined through the use of non-destructive AlamarBlue ${ }^{\circledR}$ assay as previously described (Caliari and Harley, 2011). Healthy, viable cells continuously convert the active ingredient in AlamarBlue ${ }^{\circledR}$ (resazurin) to a fluorescent byproduct (resorufin), allowing comparison of the gross metabolic activity of each cell-seeded construct. Cell-seeded scaffolds were incubated at $37{ }^{\circ} \mathrm{C}$ in alamarBlue (Invitrogen, Carlsbad, CA) solution with gentle shaking for $2 \mathrm{~h}$ (Tierney et al., 2009). Resorufin fluorescence was measured (excitation: $540 \mathrm{~nm}$, emission: $590 \mathrm{~nm}$ ) via a fluorescent spectrophotometer (Tecan, Switzerland). Relative cell metabolic activity was determined from a standard curve generated from known cell numbers prior to seeding the scaffolds and reported as a percentage of the total number of seeded cells.

\section{Measurement of cell-mediated scaffold contraction}

At days 1, 4, 7, and 14, the diameter of each scaffold disk was measured using a standard drafting template. The measurements were then normalized against the diameter of each scaffold 
at day 0 to determine cell-mediated contraction of each scaffold disk (Caliari et al., 2012; Spilker et al., 2001).

\section{Quantification of cell number}

The total number of cells on each scaffold was assayed via DNA quantification (Caliari and Harley, 2011; Kim et al., 1988). Scaffolds were rinsed in PBS and placed in a papain solution (Sigma-Aldrich, St. Louis, MO) $\left(60^{\circ} \mathrm{C}, 24\right.$ hours) to digest the scaffolds and lyse the cells. Hoechst 33258 dye (Invitrogen, Carlsbad, CA) was used to fluorescently label double-stranded DNA (Kim et al., 1988). Fluorescence was read (excitation: 360nm; emission: 465nm) via a fluorescent spectrophotometer (Tecan, Switzerland). The number of cells within the scaffolds after culture was normalized against the scaffold with the largest crosslinking density (5:2:1) and largest pore size $\left(-10^{\circ} \mathrm{C} \mathrm{T}_{\mathrm{f}}\right)$ in order to serve as a comparison to previous work (Caliari and Harley, 2011).

\section{RNA isolation and real-time PCR}

RNA from the tenocytes in the scaffolds was extracted at days 1, 4, 7 ,and 14, using an RNeasy Plant Mini kit (Qiagen, Valencia, CA) and then was reverse transcribed to cDNA in a Bio-Rad S1000 thermal cycler using the QuantiTect Reverse Transcription kit (Qiagen, Valencia, CA). as previously described (Caliari and Harley, 2011). Real-time PCR reactions were carried out in triplicate, using 10ng of cDNA and QuantiTect SYBR Green PCR kit (Qiagen, Valencia, CA) in a 7900HT Fast Real-Time PCR system (Carlsbad, CA). The primers used were consistent with previous studies (Table 2; collagen type I alpha 2; collagen type III alpha 1; cartilage oligomeric matrix protein; decorin; scleraxis; tenascin-c, C; matrix metalloproteinases 1, 3, and 13; 
glyceraldehyde 3-phosphate dehydrogenase, housekeeping) (Caliari and Harley, 2011; Caliari et al., 2012), and were synthesized by Integrated DNA Technologies (Coralville, IA). Results were generated using the $\Delta \Delta \mathrm{Ct}$ method and all results were expressed as fold changes normalized to the expression levels of cells on the $-10^{\circ} \mathrm{C} / 5: 2: 1$ scaffold group at day 1 for comparison to previous studies (Caliari and Harley, 2013; Caliari and Harley, 2011; Caliari et al., 2011; Caliari et al., 2012). The expression levels of the MMP genes are shown relative to the normalized scaffold contraction for each group at each time point in order to better elucidate the interplay of cell-mediated contraction forces and cell-mediated remodeling (Clegg et al., 2007; Gotoh et al., 1997; Jones et al., 2006; Lo et al., 2004)

\section{Statistical Analysis}

One-way analysis of variance (ANOVA) was performed on all data sets within each group to examine differences with time, while two-way ANOVAs (independent factors: crosslinking density, $\mathrm{T}_{\mathrm{f}}$ ) were applied to data sets for each time point. For scaffold contraction, significant changes from the day 0 time point were determined by Dunnett's post-hoc test. Statistical significance for all other measurements was determined by Tukey-HSD post-hoc tests. Significance was set at $p<0.05$. Cell metabolic activity $(\mathrm{n}=6)$, scaffold diameter $(\mathrm{n}=6)$, and gene expression $(n=3)$ were analyzed at each time point. For MMP gene expression relative to normalized scaffold diameter, linear or exponential regression models were used to fit the data and corresponding $R^{2}$ values ( $\left.n=27\right)$ were reported. Error was reported in figures as the standard error of the mean unless otherwise noted.

\section{Results}




\section{Tenocyte viability in CG scaffolds}

Tenocytes remained viable in all scaffold variants across the fourteen day experiment. While the metabolic activity of the tenocyte-seeded scaffolds was consistent for all scaffold groups at day 1 after seeding, by day 14 significant expansion in overall metabolic activity and cell number was observed. Here, cell number and construct metabolic activity increased significantly $(p<0.01)$ with crosslinking density, with the largest increases in cell number and metabolic activity seen for the most strongly crosslinked (EDC:NHS:COOH 5:2:1) variants (Figure 1A, 1B). A significant effect of both crosslinking density $(p<0.001)$ and scaffold pore size $\left(\mathrm{T}_{\mathrm{f}}, p<0.001\right)$ was observed on tenocyte mediated scaffold contraction (Figure 1C). Scaffolds crosslinked at the lightest (1:1:5) density started showing signs of contraction as early as day 1 , then showed significantly $(p<0.01)$ greater contraction (smaller normalized diameter) than scaffolds crosslinked at higher densities on all following days. Scaffolds crosslinked at a middle (5:2:5) density began to show signs on contraction by day 4, and on all following days were significantly more contracted than the scaffolds crosslinked at the highest (5:2:1) density, which exhibited little-to-no contraction throughout the 14-day experiment. For crosslinking densities that yielded significant contraction $(1: 1: 5,5: 2: 5)$, there was also a significant $(p<0.01)$ effect of scaffold pore size on contraction. Scaffolds with larger pore sizes (higher $\mathrm{T}_{\mathrm{f}}$ ) showed greater contraction when compared to scaffolds with the same crosslinking density but smaller pore sizes.

\section{Tenocyte gene expression within CG scaffolds}

We subsequently examined the gene expression profiles of the cells remaining in each construct after 14 days in culture. Three distinct sub-sets of genes were examined: structural proteins 
associated with tendon ECM (COL1A2, COL3A1, COMP, DCN; Figure 2); genes associated with tendon phenotype (SCX, TNC; Figure 3); and genes associated with matrix remodeling (MMP1, MMP3, MMP13; Figure 4).

\section{Structural protein gene expression}

Gene expression levels of COL1A1, COL3A3, COMP, and DNC showed some global changes in expression for earlier time points (days 1, 4, and 7; Supplementary Figures 1, 2). By day 4, the scaffolds least resistant to contraction (1:1:5 crosslinking density) showed significant increases in COL3A1, COMP, and DCN, while scaffolds moderately able to resist contraction (5:2:5 crosslinking density) showed significantly higher expression of all four structural protein markers and scaffolds most resistant to contraction (5:2:1 crosslinking density) only showed increased expression of $D C N$. More interestingly, we examined changes in structural genes at day 14, at which point significant cell-mediated contraction had occurred (Figure 2). Notably, scaffolds most resistant to contraction $\left(5: 2: 1\right.$ crosslinking density; $-40^{\circ} \mathrm{C}$ and $\left.-60^{\circ} \mathrm{C} \mathrm{T}_{\mathrm{f}}\right)$ showed significantly higher expression of COL1A2 compared to scaffolds with lower crosslinking densities and the same $\mathrm{T}_{\mathrm{f}}$ (Figure 2). Further, while not always significant an overall change in expression profiles for all genes were observed, with crosslinking groups less resistant to contraction (1:1:5, 5:2:5) showing decreases in expression with decreasing pore size while the crosslinking groups most resistant to contraction (5:2:1) showing increasing in expression with decreasing pore size. Finally, while there were no significant differences in expression of $D C N$, the scaffold with the smallest pores most resistant to contraction (-60 ${ }^{\circ} \mathrm{C} \mathrm{T}_{\mathrm{f}} ; 5: 2: 1$ crosslinking) showed significantly higher expression of COMP than all other scaffold groups at day 14 (Figure 2). 


\section{Tendon phenotype gene expression}

Expression of the transcription factor SCX increased initially in all scaffold variants (through day 4). However, significant cell-mediated contraction began to affect the expression profile for subsequent days for the scaffold groups least resistant to contraction (1:1:5 and 5:2:5 crosslinking density) while the scaffold group resistant to contraction (5:2:1 crosslinking density) maintained the elevated SCX expression through days 14 (Supplementary Figure 3). Comparing between scaffold pore size and crosslinking at day 14, we observed the scaffold variant most resistant to contraction $\left(5: 2: 1\right.$ crosslinking density, $\left.-60^{\circ} \mathrm{C} \mathrm{T}_{\mathrm{f}}\right)$ showed significantly higher SCX expression than all other groups. Further scaffolds with reduced resistance to contraction (1:1:5 crosslinking density, $\left.-40^{\circ} \mathrm{T}_{\mathrm{f}}\right)$ showed significantly lower expression of $S C X$ than all other groups (Figure 3). TNC expression followed a similar trend to SCX, with all groups showing increased expression though day 7 (Supplementary Figure 3). While not significant by day 14, an increasing trend in TNC expression was observed for scaffolds most resistant to contraction (5:2:1 crosslinking density; -40 and $\left.-60^{\circ} \mathrm{C} \mathrm{T}_{\mathrm{f}}\right)$.

\section{MMP gene Expression}

All three MMP genes examined (MMP1, MMP3, and MMP13) showed similar trends regarding expression between groups and over time (Supplementary Figure 4). In general, MMP expression levels increased with time point and were significantly higher in the scaffolds least resistant to contraction (warmer $\mathrm{T}_{\mathrm{f}}$, lower crosslinking densities). More interestingly, we subsequently examined MMP expression profiles against the normalized (versus starting diameter) diameter of each scaffold, collapsing the effect of time, pore size, and crosslinking 
density onto a single plot (Figure 4). With the exception of day 1 at which point minimal contraction had taken place $\left(\mathrm{R}^{2}=0.25\right)$, we observed very strong correlation between increasing MMP expression profiles and increased scaffold contraction, with $\mathrm{R}^{2} \geq 0.84$ for day 14 results. Interestingly, MMP1 and MMP3 showed effectively linear increases in expression with scaffold crosslinking and time, while MMP13 showed an exponential increase in expression with time. Scaffolds least resistant to contraction (1:1:5 density, $\left.-10^{\circ} \mathrm{C} \mathrm{T}_{\mathrm{f}}\right)$ showed more than 600 fold increase in MMP13 over the course of 14 days (Figure 4C).

\section{Discussion}

Biomaterial scaffold mechanical properties at both the macro and micro-scales have been widely reported to influence cellular behaviors such as adhesion, migration, proliferation, and differentiation (Engler et al., 2004; Freyman et al., 2001; Grinnell et al., 2003; Pelham and Wang, 1997; Peyton and Putnam, 2005; Yeung et al., 2005; Zaman et al., 2006). The motivation for this work is the significant de-differentiation often observed with tenocytes when culture in vitro as well as the observation that structural alignment in two-dimensions and application of tensile strain may reduce this de-differentiation (Taylor et al., 2009). Future tendon tissue engineering efforts require the expansion of primary tenocytes in order to address large injuries, necessitating approaches that use three-dimensional biomaterial constructs for cell culture and eventual in vivo implantation. If a microstructurally-aligned material is unable to maintain a high degree of anisotropy due to contractile forces, the microstructural cues that guide cell-fate decisions will be significantly altered as the cells remodel the local microenvironment. 
Here we describe the production and analysis of a series of anisotropic CG scaffolds fabricated at different $\mathrm{T}_{\mathrm{f}}$ and a range of crosslinking densities for the in vitro culture of primary equine tenocytes. Our group recently described an approach where the relative density of a series of CG scaffolds was varied in order to prevent TC-mediated contraction, resulting in reduced scaffold contraction, maintenance of the aligned structural cues provided by the scaffold and resultant increased maintenance of TC-associated gene expression profiles for cells within the scaffold (Caliari et al., 2012). However, changes in the relative density of the scaffold result in changes in scaffold pore size as well as in the mechanical properties of the scaffold at the scale of the overall construct and at the level of an individual cell within the scaffold. This observation therefore motivated our effort here to generate a wider library of CG scaffolds to more specifically examine the effect of scaffold architecture on resultant TC-mediated contraction and phenotypic stability.

Low-density open-cell foams such as the CG scaffold demonstrate a unique set of structuremechanic relationships (Gibson et al., 2010). For these materials it is essential to consider the mechanical property at the scale of the overall construct (bulk properties) as well as at the level of the individual fibers (termed struts) that make up the scaffold architecture and to which individual cells attach. The bulk mechanical properties of the scaffold depend critically on the crosslinking density and relative density ( 1 - porosity), not pore size, of the scaffold. For example, the elastic modulus of the bulk scaffold $\left(E^{*}\right)$ depends on the crosslinking density of the collagen (defining $E_{s}$, the modulus of the individual strut) along with the relative density $\left(\rho^{*} / \rho_{s}\right)$ of the scaffold: $E^{*} \sim E_{s^{*}}\left(\rho^{*} / \rho_{s}\right)^{2}$ (Harley et al., 2007c). However, modulus is insensitive to scaffold pore size (Gibson and Ashby, 1997; Harley et al., 2007c). However, cell-mediated 
contraction is mediated not by the bulk elastic properties, but rather the mechanical properties of the individual struts to which they attach. Previous studies have shown that contractile cells possess the ability to generate sufficient contractile forces to buckle individual struts and deform the local pore structure around them (Freyman et al., 2001). These local contractions, when compounded throughout the entire scaffold result in a macroscopic deformation of the overall scaffold. Further, the magnitude of contractile forces generated by individual cells can be estimated via the flexural rigidity of the strut, which depends on crosslinking density $\left(E_{s}\right)$ and also the length and thickness of the strut (Harley et al., 2007a). Here, increasing the pore size for a series of scaffolds with a set relative density increases the length of individual struts (Harley et al., 2007c). Therefore, selective modification to both scaffold crosslinking and pore size provides a wider platform to consider the influence of local strut mechanical properties, resistance to TCmediated contraction, and subsequent maintenance of tenogenic gene expression profiles within the scaffold.

For this project we employed a previously defined set of scaffold fabrication techniques, altering the freezing temperature during lyophilization to generate a series of anisotropic scaffolds within decreasing pore size $\left(-10^{\circ} \mathrm{C},-40^{\circ} \mathrm{C},-60^{\circ} \mathrm{C} ; 243,152\right.$, and $55 \mu \mathrm{m}$ pore size) (Caliari and Harley, 2011). We subsequently employed a series of three discrete carbodiimide crosslinking densities (1:1:5, 5:2:5, 5:2:1) that span a 3.6-fold increase in stiffness (Harley et al., 2007c). TC-mediated contraction, metabolic activity and cell number, and resultant changes in gene expression profiles for a diverse set of tendon-associated structural proteins, tenocyte markers, and matrixremodeling genes were then traced across the full set of 9 experimental scaffold groups. Here, 
we observed scaffold pore size and crosslinking density were able to elicit differential responses during long-term cell culture.

In general, an increase in the number and metabolic activity of primary equine tenocytes was observed with culture time. However, we noted a significant effect of both crosslinking density and scaffold pore size on cell-mediated contraction of the scaffolds (Figure 1A). Not surprisingly, increasing scaffold crosslinking density resulted in reduced cell-mediated contraction; however, we also noted a significant effect of pore size on contraction, with scaffolds containing smaller pores more able to resist cellular contractile forces over time (Figure 1C). The loss of structural stability via contraction has a significant negative effect on cell metabolic activity due with time, likely due to reduced biotransport as a result of the more contracted scaffold structure. Applied compressive loading has previously been shown to alter bulk scaffold permeability (O'Brien et al., 2007; Weisgerber et al., 2013), suggesting the need for improved technologies to locally monitor scaffold permeability as a result of localized cellcontraction. So while we observed a significant reduction in metabolic activity and cell number after 14 days of culture in scaffolds that exhibited significant contraction (Figure 1A, 1B), it was important to establish the effect of this contraction on the phenotype of the resultant cells.

We subsequently examined the effects of cell-mediated contraction on the maintenance of a healthy tendon-like phenotype via transcript levels of ECM proteins associated with native tendon (COL1A2, COL3A1, COMP, DCN) (Lui et al., 2011) as well as transcription factors and matricellular markers of tenocytes (SCX, TNC) (Blitz et al., 2009; Doroski et al., 2010; Pryce et al., 2009; Schweitzer et al., 2010) (Figures 2-3). Lastly, we also examined a series of matrix 
metalloproteinases (MMP1, MMP3, MMP13) important for cell migration, wound healing, and matrix remodeling, but also whose reduced expression levels are indicative of healthy tendon (Clegg et al., 2007; Gotoh et al., 1997; Jones et al., 2006). Transcript levels at each time point were normalized against expression levels of tenocytes in scaffolds with a $-10^{\circ} \mathrm{C} \mathrm{T}_{\mathrm{f}}$ and 5:2:1 crosslinking density to compare to previous studies investigating the influence of the CG scaffold microenvironment on the transcriptomic stability of tenocytes (Caliari and Harley, 2013; Caliari and Harley, 2011; Caliari et al., 2012). Importantly, by day 14, COL1A2, COL3A1, COMP, and DCN all showed decreased expression in scaffolds with reduced crosslinking 1:1:5 and 5:2:5 scaffolds and with the largest pore size (least resistant to contraction). Expression levels were increased in scaffolds most resistant to contraction $\left(T_{f}-60 ; 5: 2: 1\right.$ crosslinking density). Expression of $S C X$ was significantly higher in scaffolds most resistant to contraction $\left(\mathrm{T}_{\mathrm{f}}\right.$ -60; 5:2:1 crosslinking density) while TNC expression following a similar, but not significant, trend. Further, heightened MMP1, MMP3, and MMP13 expression all followed nearly identical and dramatic trends. Most interestingly, plotting the results not as a function of scaffold pore size or crosslinking density, but rather versus overall resistance to TC-mediated contraction, these data collapse onto clear trends of reduced TC-associated markers with increasing cell-mediated contraction (Figure 4).

While our earlier results suggested reduced TC-associated markers with increasing scaffold contraction as a result in changes in overall scaffold relative density (Caliari et al., 2012), changes in relative density masked our ability to determine whether the stiffness of the scaffold strut versus the flexural rigidity of the strut was the primary design consideration that could be used in subsequent biomaterial variants. Here, by selectively modifying scaffold pore size and 
crosslinking density, we are able to independently tune strut length versus strut stiffness. Interestingly, we found that the overall ability to resist contraction was the best predictor of maintenance of tenocyte-associated gene expression profiles. However, examining the effect of pore size versus crosslinking in more detail, we note that crosslinking density provides a primary means to resist TC-mediated contraction. For lower crosslinking densities (1:1:5, 5:2:5) where significant contraction was observed, the poorest response was seen in the smallest pore size variants, likely due to the significant contraction not only destroying the aligned structure signals provided by the pore, but also significantly reducing biotransport. However, in the highest crosslinking density (5:2:1) where limited contraction was observed, the scaffold with the smallest pore size more resistant to contraction demonstrated the highest level of TC-associated genes. Together, these results suggest that while scaffold ability to resist TC-mediated contraction forces play a significant role in regulating tenocyte gene expression, increasingly scaffold crosslinking, not necessarily changes in pore size, provide the primary means to optimize culture conditions for expansion and maintenance of primary tenocytes. While future investigations will be required to further optimize the three-dimensional scaffold most appropriate for long-term tenocyte culture, findings here provide critical insight as to the use of crosslinking versus structural changes in the scaffold to accomplish this goal.

\section{Conclusions}

This work describes the evaluation of the ability for a series of anisotropic CG scaffolds to promote the expansion of primary tenocytes in vitro. In general, equine tenocytes showed increased proliferation and metabolic activity as well as less cell-mediated contraction in scaffolds with higher crosslinking densities and smaller pore sizes. Gene expression analysis 
showed scaffolds most resistant of cell-mediated contraction were able to maintain a tendon-like-

phenotype over the course of the experiment. Importantly, the high expression levels of MMPs 1 ,

3 , and 13 in scaffolds that showed more contraction indicate a high degree of scaffold

remodeling and loss of tendon-like phenotype. This suggests that the structural stability of

biomaterials for tissue engineering applications is of particular importance when considering

clinical translation.

\section{Acknowledgements}

The authors would like to acknowledge Dr. Allison Stewart (Veterinary Sciences, UIUC) for providing equine tendon cells and the IGB Core Facilities for the use of their real-time PCR system. Research reported in this publication was supported by the National Institute of Arthritis and Musculoskeletal and Skin Diseases of the National Institutes of Health under Award Numbers R03 AR062811 and R21 AR063331. We are grateful for funding provided by the Chemistry-Biology Interface Training Program NIH NIGMS T32GM070421 (WKG). EMI was funded through a Research Experience for Undergraduates program within the National Science Foundation (NSF) Science and Technology Center Emergent Behavior of Integrated Cellular Systems (EBICS) Grant CBET-0939511. Additional support was provided by the Chemical and Biomolecular Engineering Dept. and the Carl R. Woese Institute for Genomic Biology (BACH) at the University of Illinois at Urbana-Champaign. This research was carried out in part in the Frederick Seitz Materials Research Laboratory Central Facilities, University of Illinois, which are partially supported by the U.S. Department of Energy under grants DE-FG02-07ER46453 and DE-FG02-07ER46471. 


\section{Tables}

Table 1. Effect of fabrication and crosslinking on scaffold structural and mechanical properties. This table summarizes previously reported data on pore size (Caliari and Harley, 2011) and the effect of crosslinking on elastic modulus (Harley et al., 2007b) and swelling ratio (Vickers et al., 2010).

\begin{tabular}{ll|ll}
\hline $\mathbf{T}_{\mathbf{f}}{ }^{\mathbf{0}} \mathbf{C}$ & Transverse pore size, $\boldsymbol{\mu m}$ & EDC crosslinking & Relative stiffness, $\boldsymbol{E} / \boldsymbol{E}_{\boldsymbol{D H T}}$ \\
\hline-10 & $242.7 \pm 28.8$ & DHT (standard) & $1.0 \pm 0.051\left(\mathrm{E}_{\mathrm{DHT}}=208 \pm 41 \mathrm{~Pa}\right)$ \\
-40 & $152.4 \pm 25.1$ & $1: 1: 5$ & $2.0 \pm 0.11$ \\
-60 & $55.3 \pm 17.6$ & $5: 2: 1$ & $7.20 \pm 0.14$ \\
\hline
\end{tabular}

Table 2. PCR primer sequences.

\begin{tabular}{|c|c|c|}
\hline Transcript & Sequence & Reference \\
\hline COL1A2 & $\begin{array}{l}\text { Forward: 5'- GCACATGCCGTGACTTGAGA -3' } \\
\text { Reverse: 5'- CATCCATAGTGCATCCTTGATTAGG -3' }\end{array}$ & (Taylor et al., 2009) \\
\hline COL3A1 & $\begin{array}{l}\text { Forward: 5’- GTCCACCTGAGGAACTGTCT--3’' } \\
\text { Reverse: 5’- TGATCAGGACCACCAACATCA-3' }\end{array}$ & (Taylor et al., 2009) \\
\hline СOMP & $\begin{array}{l}\text { Forward: 5’- GGTGCGGCTGCTATGGAA -3’' } \\
\text { Reverse: 5'- CCAGCTCAGGGCCCTCAT -3’’ }\end{array}$ & (Taylor et al., 2009) \\
\hline$D C N$ & $\begin{array}{l}\text { Forward: 5'- CATCCAGGTTGTCTACCTTCATAACA-3' } \\
\text { Reverse: 5'- CCAGGTGGGCAGAAGTCATT -3' }\end{array}$ & (Taylor et al., 2009) \\
\hline$S C X$ & $\begin{array}{l}\text { Forward: 5'- TCTGCCTCAGCAACCAGAGA -3’' } \\
\text { Reverse: 5'- TCCGAATCGCCGTCTTTC-3’' }\end{array}$ & (Taylor et al., 2009) \\
\hline TNC & $\begin{array}{l}\text { Forward: 5'- GGGCGGCCTGGAAATG -3' } \\
\text { Reverse: 5'- CAGGCTCTAACTCCTGGATGATG-3' }\end{array}$ & (Taylor et al., 2009) \\
\hline$M M P 1$ & $\begin{array}{l}\text { Forward: 5'- GGTGAAGGAAGGTCAAGTTCTGAT -3' } \\
\text { Reverse: 5'- AGTCTTCTACTTTGGAAAAGAGCTTCT -3' }\end{array}$ & (Garvican et al., 2008) \\
\hline MMP3 & $\begin{array}{l}\text { Forward: 5’- GCACATGCCGTGACTTGAGA-3' } \\
\text { Reverse: 5’- CCTATGGAAGGTGACTCCATGTG -3’' }\end{array}$ & (Garvican et al., 2008) \\
\hline MMP13 & $\begin{array}{l}\text { Forward: 5'- CTGGAGCTGGGCACCTACTG -3' } \\
\text { Reverse: 5'- ATTTGCCTGAGTCATTATGAACAAGAT-3' }\end{array}$ & (Garvican et al., 2008) \\
\hline$G A P D H$ & $\begin{array}{l}\text { Forward: 5’- GCATCGTGGAGGGACTCA -3' } \\
\text { Reverse: 5'- GCCACATCTTCCCAGAGG-3’' }\end{array}$ & (Taylor et al., 2009) \\
\hline
\end{tabular}




\section{Figure Captions}

Figure 1. Tenocyte viability and contraction of CG scaffolds. (A) Metabolic activity of tenocytes at days 1 and 14 for all nine CG scaffold groups. (B) Normalized number of cells at day 14 for all nine CG scaffold groups (all scaffolds normalized vs. control scaffold with the largest pore size and highest crosslinking density). (C) Scaffold contraction, normalized to the initial scaffold diameter, at days 1, 4, 7, and 14 for all nine CG scaffold groups. Data expressed as mean \pm SEM $(n=6)$. $\vee$ : significantly $(p<0.01)$ lower than initial scaffold diameter. $\dagger$ : significantly $(p<0.01)$ different than scaffolds of same pore size with lowest $(1: 1: 5)$ crosslinking density. $\ddagger$ : significantly $(p<0.01)$ different than scaffolds of same pore size with middle (5:2:5) crosslinking density. ${ }^{*}$ : significantly $(p<0.01)$ different from scaffolds of same crosslinking density with largest pore size $\left(-10^{\circ} \mathrm{C} \mathrm{T}_{\mathrm{f}}\right)$. \#: significantly $(p<0.01)$ different from scaffolds of same crosslinking density with middle pore size $\left(-40^{\circ} \mathrm{C} \mathrm{T}_{\mathrm{f}}\right) .<<$ FORMAT: 2 column figure $>>$

Figure 2. Structural protein gene expression. Expression levels of (A) COL1A2, (B) COL3A1, (C) $C O M P$, and (D) DCN after 14 days in all 9 scaffold groups. Data expressed as mean \pm SEM $(n=3) . \dagger$ : significantly $(p<0.01)$ different than scaffolds of same pore size with lowest (1:1:5) crosslinking density. ¥: significantly $(p<0.01)$ different than scaffolds of same pore size with middle (5:2:5) crosslinking density. *: significantly $(p<0.01)$ different from scaffolds of same crosslinking density with largest pore size $\left(-10^{\circ} \mathrm{C} \mathrm{T}_{\mathrm{f}}\right)$. \#: significantly $(p<0.01)$ different from scaffolds of same crosslinking density with middle pore size $\left(-40^{\circ} \mathrm{C} \mathrm{T}_{\mathrm{f}}\right) .<<$ FORMAT: 2 column figure $>>$

Figure 3. Tendon phenotype gene expression. Expression levels of (A) SCX and (B) TNC after 14 days in all 9 scaffold groups. Data expressed as mean \pm SEM $(n=3)$. $\uparrow$ : significantly $(p<0.01)$ different than scaffolds of same pore size with lowest $(1: 1: 5)$ crosslinking density. $\ddagger$ : significantly $(p<0.01)$ different than scaffolds of same pore size with middle (5:2:5) crosslinking density. ${ }^{*}$ : significantly $(p<0.01)$ different from scaffolds of same crosslinking density with largest pore size $\left(-10^{\circ} \mathrm{C} \mathrm{T}_{\mathrm{f}}\right)$. \#: significantly $(p$ $<0.01)$ different from scaffolds of same crosslinking density with middle pore size $\left(-40^{\circ} \mathrm{C} \mathrm{T}_{\mathrm{f}}\right)$. $<<$ FORMAT: 2 column figure $>>$

Figure 4. MMP gene expression. (A) MMP 1, (B) MMP3, and (C) MMP13 expression levels at days 1, 4, 7, and 14 relative to normalized scaffold diameter for all 9 scaffold groups. Data expressed as mean \pm SEM $(n=3)$. The degree of correlation $\left(\mathrm{R}^{2}\right)$ was between gene expression and scaffold contraction was determined as a function of experimental time point (day 1, 4, 7, and 14). <<FORMAT: 1 column figure >> 


\section{References}

Blitz, E., Viukov, S., Sharir, A., Shwartz, Y., Galloway, J.L., Pryce, B.A., Johnson, R.L., Tabin, C.J., Schweitzer, R., Zelzer, E., 2009. Bone ridge patterning during musculoskeletal assembly is mediated through SCX regulation of Bmp4 at the tendon-skeleton junction. Dev Cell 17, 861873.

Breidenbach, A.P., Gilday, S.D., Lalley, A.L., Dyment, N.A., Gooch, C., Shearn, J.T., Butler, D.L., 2013. Functional tissue engineering of tendon: Establishing biological success criteria for improving tendon repair. Journal of biomechanics.

Butler, D.L., Juncosa-Melvin, N., Boivin, G.P., Galloway, M.T., Shearn, J.T., Gooch, C., Awad, H., 2008. Functional tissue engineering for tendon repair: A multidisciplinary strategy using mesenchymal stem cells, bioscaffolds, and mechanical stimulation. J Orthop Res 26, 1-9. Caliari, S.R., Harley, B.A., 2013. Composite Growth Factor Supplementation Strategies to Enhance Tenocyte Bioactivity in Aligned Collagen-GAG Scaffolds. Tissue Eng Part A. Caliari, S.R., Harley, B.A.C., 2011. The effect of anisotropic collagen-GAG scaffolds and growth factor supplementation on tendon cell recruitment, alignment, and metabolic activity. Biomaterials 32, 5330-5340.

Caliari, S.R., Ramirez, M.A., Harley, B.A.C., 2011. The development of collagen-GAG scaffoldmembrane composites for tendon tissue engineering. Biomaterials 32, 8990-8998.

Caliari, S.R., Weisgerber, D.W., Ramirez, M.A., Kelkhoff, D.O., Harley, B.A.C., 2012. The influence of collagen-glycosaminoglycan scaffold relative density and microstructural anisotropy on tenocyte bioactivity and transcriptomic stability. Journal of the Mechanical Behavior of Biomedical Materials 11, 27-40.

Caporali, E., Kapoor, A., Kenis, P.A., Stewart, M.C., 2009. TGF- $\beta$ and microtopographical cues promote expression of tenogenic marker genes and tenocyte alignment., Proceedings of the $36^{\text {th }}$ Annual Conference of the Veterinary Orthopedic Society.

Clegg, P.D., Strassburg, S., Smith, R.K., 2007. Cell phenotypic variation in normal and damaged tendons. International Journal of Experimental Pathology 88, 227-235.

Doroski, D.M., Levenston, M.E., Temenoff, J.S., 2010. Cyclic tensile culture promotes fibroblastic differentiation of marrow stromal cells encapsulated in poly(ethylene glycol)-based hydrogels. Tissue Eng Part A 16, 3457-3466.

Engler, A., Bacakova, L., Newman, C., Hategan, A., Griffin, M., Discher, D., 2004. Substrate compliance versus ligand density in cell on gel responses. Biophys J 86, 617-628.

Farrell, E., O'Brien, F.J., Doyle, P., Fischer, J., Yannas, I., Harley, B.A., O'Connell, B., Prendergast, P.J., Campbell, V.A., 2006. A collagen-glycosaminoglycan scaffold supports adult rat mesenchymal stem cell differentiation along osteogenic and chondrogenic routes. Tissue engineering 12, 459-468.

Fox, A.J., Bedi, A., Deng, X.H., Ying, L., Harris, P.E., Warren, R.F., Rodeo, S.A., 2011. Diabetes mellitus alters the mechanical properties of the native tendon in an experimental rat model. J Orthop Res 29, 880-885.

Freyman, T.M., Yannas, I.V., Yokoo, R., Gibson, L.J., 2001. Fibroblast contraction of a collagen-GAG matrix. Biomaterials 22, 2883-2891.

Garvican, E.R., Vaughan-Thomas, A., Redmond, C., Clegg, P.D., 2008. Chondrocytes harvested from osteochondritis dissecans cartilage are able to undergo limited in vitro chondrogenesis despite having perturbations of cell phenotype in vivo. Journal of Orthopaedic Research 26, 1133-1140. 
Gibson, L.J., Ashby, M.F., 1997. Cellular solids: structure and properties, 2nd ed. Cambridge University Press, Cambridge, U.K.

Gibson, L.J., Ashby, M.F., Harley, B.A., 2010. Cellular materials in nature and medicine. Cambridge University Press, Cambridge ; New York.

Gotoh, M., Hamada, K., Yamakawa, H., Tomonaga, A., Inoue, A., Fukuda, H., 1997.

Significance of granulation tissue in torn supraspinatus insertions: An immunohistochemical

study with antibodies against interleukin-1 $\beta$, cathepsin D, and matrix metalloprotease-1. Journal of Orthopaedic Research 15, 33-39.

Grinnell, F., Ho, C.H., Tamariz, E., Lee, D.J., Skuta, G., 2003. Dendritic fibroblasts in threedimensional collagen matrices. Mol Biol Cell 14, 384-395.

Harley, B.A., Freyman, T.M., Wong, M.Q., Gibson, L.J., 2007a. A new technique for calculating individual dermal fibroblast contractile forces generated within collagen-GAG scaffolds.

Biophys J 93, 2911-2922.

Harley, B.A., Kim, H.D., Zaman, M.H., Yannas, I.V., Lauffenburger, D.A., Gibson, L.J., 2008.

Microarchitecture of three-dimensional scaffolds influences cell migration behavior via junction interactions. Biophys J 95, 4013-4024.

Harley, B.A., Leung, J.H., Silva, E.C., Gibson, L.J., 2007b. Mechanical characterization of collagen-glycosaminoglycan scaffolds. Acta Biomater 3, 463-474.

Harley, B.A., Leung, J.H., Silva, E.C.C.M., Gibson, L.J., 2007c. Mechanical characterization of collagen-glycosaminoglycan scaffolds. Acta Biomaterialia 3, 463-474.

Harley, B.A., Spilker, M.H., Wu, J.W., Asano, K., Hsu, H.P., Spector, M., Yannas, I.V., 2004.

Optimal degradation rate for collagen chambers used for regeneration of peripheral nerves over long gaps. Cells Tissues Organs 176.

James, R., Kesturu, G., Balian, G., Chhabra, A.B., 2008. Tendon: biology, biomechanics, repair, growth factors, and evolving treatment options. J Hand Surg Am 33, 102-112.

Jones, G.C., Corps, A.N., Pennington, C.J., Clark, I.M., Edwards, D.R., Bradley, M.M., Hazleman, B.L., Riley, G.P., 2006. Expression profiling of metalloproteinases and tissue inhibitors of metalloproteinases in normal and degenerate human achilles tendon. Arthritis \& Rheumatism 54, 832-842.

Kapoor, A., Caporali, E.H.G., Kenis, P.J.A., Stewart, M.C., 2010. Microtopographically patterned surfaces promote the alignment of tenocytes and extracellular collagen. Acta Biomaterialia 6, 2580-2589.

Kim, Y.-J., Sah, R.L.Y., Doong, J.-Y.H., Grodzinsky, A.J., 1988. Fluorometric assay of DNA in cartilage explants using Hoechst 33258. Analytical Biochemistry 174, 168-176.

Liu, Y., Ramanath, H.S., Wang, D.A., 2008. Tendon tissue engineering using scaffold enhancing strategies. Trends Biotechnol 26, 201-209.

Lo, I.K.Y., Marchuk, L.L., Hollinshead, R., Hart, D.A., Frank, C.B., 2004. Matrix

Metalloproteinase and Tissue Inhibitor of Matrix Metalloproteinase mRNA Levels Are Specifically Altered in Torn Rotator Cuff Tendons. The American Journal of Sports Medicine 32, 1223-1229.

Lui, P.P.Y., Rui, Y.F., Ni, M., Chan, K.M., 2011. Tenogenic differentiation of stem cells for tendon repair-what is the current evidence? Journal of Tissue Engineering and Regenerative Medicine 5, e144-e163.

Maeda, E., Shelton, J.C., Bader, D.L., Lee, D.A., 2009. Differential regulation of gene expression in isolated tendon fascicles exposed to cyclic tensile strain in vitro. J Appl Physiol 106, 506-512. 
O'Brien, F.J., Harley, B.A., Waller, M.A., Yannas, I.V., Gibson, L.J., Prendergast, P.J., 2007. The effect of pore size on permeability and cell attachment in collagen scaffolds for tissue engineering. Technol Health Care 15, 3-17.

O'Brien, F.J., Harley, B.A., Yannas, I.V., Gibson, L.J., 2005. The effect of pore size on cell adhesion in collagen-GAG scaffolds. Biomaterials 26, 433-441.

O’Brien, F.J., Harley, B.A., Yannas, I.V., Gibson, L., 2004. Influence of freezing rate on pore structure in freeze-dried collagen-GAG scaffolds. Biomaterials 25, 1077-1086.

Olde Damink, L.H.H., Dijkstra, P.J., van Luyn, M.J.A., van Wachem, P.B., Nieuwenhuis, P., Feijen, J., 1996. Cross-linking of dermal sheep collagen using a water-soluble carbodiimide. Biomaterials 17, 765-773.

Paxton, J.Z., Hagerty, P., Andrick, J.J., Baar, K., 2011. Optimizing an Intermittent Stretch Paradigm Using ERK1/2 Phosphorylation Results in Increased Collagen Synthesis in Engineered Ligaments. Tissue Eng Part A.

Pelham, R.J., Wang, Y.-l., 1997. Cell locomotion and focal adhesions are regulated by substrate flexibility. Proceedings of the National Academy of Sciences 94, 13661-13665. Peyton, S.R., Putnam, A.J., 2005. Extracellular matrix rigidity governs smooth muscle cell motility in a biphasic fashion. Journal of Cellular Physiology 204, 198-209.

Pryce, B.A., Watson, S.S., Murchison, N.D., Staverosky, J.A., Dunker, N., Schweitzer, R., 2009. Recruitment and maintenance of tendon progenitors by TGFbeta signaling are essential for tendon formation. Development 136, 1351-1361.

Schulz Torres, D., M. Freyman, T., Yannas, I.V., Spector, M., 2000. Tendon cell contraction of collagen-GAG matrices in vitro: effect of cross-linking. Biomaterials 21, 1607-1619.

Schweitzer, R., Zelzer, E., Volk, T., 2010. Connecting muscles to tendons: tendons and musculoskeletal development in flies and vertebrates. Development 137, 2807-2817. Spilker, M.H., Asano, K., Yannas, I.V., Spector, M., 2001. Contraction of collagenglycosaminoglycan matrices by peripheral nerve cells in vitro. Biomaterials 22, 1085-1093. Taylor, S.E., Vaughan-Thomas, A., Clements, D.N., Pinchbeck, G., Macrory, L.C., Smith, R.K., Clegg, P.D., 2009. Gene expression markers of tendon fibroblasts in normal and diseased tissue compared to monolayer and three dimensional culture systems. BMC Musculoskelet Disord 10, 27.

Tierney, C.M., Jaasma, M.J., O'Brien, F.J., 2009. Osteoblast activity on collagen-GAG scaffolds is affected by collagen and GAG concentrations. Journal of Biomedical Materials Research Part A 91A, 92-101.

Towler, D.A., Gelberman, R.H., 2006. The alchemy of tendon repair: a primer for the (S)mad scientist. J Clin Invest 116, 863-866.

Vickers, S.M., Gotterbarm, T., Spector, M., 2010. Cross-linking affects cellular condensation and chondrogenesis in type II collagen-GAG scaffolds seeded with bone marrow-derived mesenchymal stem cells. J Orthop Res 28, 1184-1192.

Weisgerber, D.W., Kelkhoff, D.O., Caliari, S.R., Harley, B.A.C., 2013. The impact of discrete compartments of a multi-compartment collagen-GAG scaffold on overall construct biophysical properties. J Mech Behav Biomed Mater 28, 26-36.

Xu, Y., Murrell, G.A., 2008. The basic science of tendinopathy. Clin Orthop Relat Res 466, 1528-1538.

Yannas, I.V., 2001. Tissue and organ regeneration in adults. Springer, New York. 
Yannas, I.V., Lee, E., Orgill, D.P., Skrabut, E.M., Murphy, G.F., 1989. Synthesis and characterization of a model extracellular matrix that induces partial regeneration of adult mammalian skin. Proc Natl Acad Sci U S A 86, 933-937.

Yeung, T., Georges, P.C., Flanagan, L.A., Marg, B., Ortiz, M., Funaki, M., Zahir, N., Ming, W., Weaver, V., Janmey, P.A., 2005. Effects of substrate stiffness on cell morphology, cytoskeletal structure, and adhesion. Cell Motility and the Cytoskeleton 60, 24-34.

Zaman, M.H., Trapani, L.M., Sieminski, A.L., MacKellar, D., Gong, H., Kamm, R.D., Wells, A., Lauffenburger, D.A., Matsudaira, P., 2006. Migration of tumor cells in 3D matrices is governed by matrix stiffness along with cell-matrix adhesion and proteolysis. Proceedings of the National Academy of Sciences 103, 10889-10894. 
pore size

Figure 1

stiffness $\begin{array}{llll}\square 1: 1: 5\left(-10^{\circ} \mathrm{C}\right) & \square 1: 1: 5\left(-40^{\circ} \mathrm{C}\right) & \square 1: 1: 5\left(-60^{\circ} \mathrm{C}\right) \\ & \square 5: 2: 5\left(-10^{\circ} \mathrm{C}\right) & \square 5: 2: 5\left(-40^{\circ} \mathrm{C}\right) & \square 5: 2: 5\left(-60^{\circ} \mathrm{C}\right) \\ \square 5: 2: 1\left(-10^{\circ} \mathrm{C}\right) & \square 5: 2: 1\left(-40^{\circ} \mathrm{C}\right) & \square 5: 2: 1\left(-60^{\circ} \mathrm{C}\right)\end{array}$
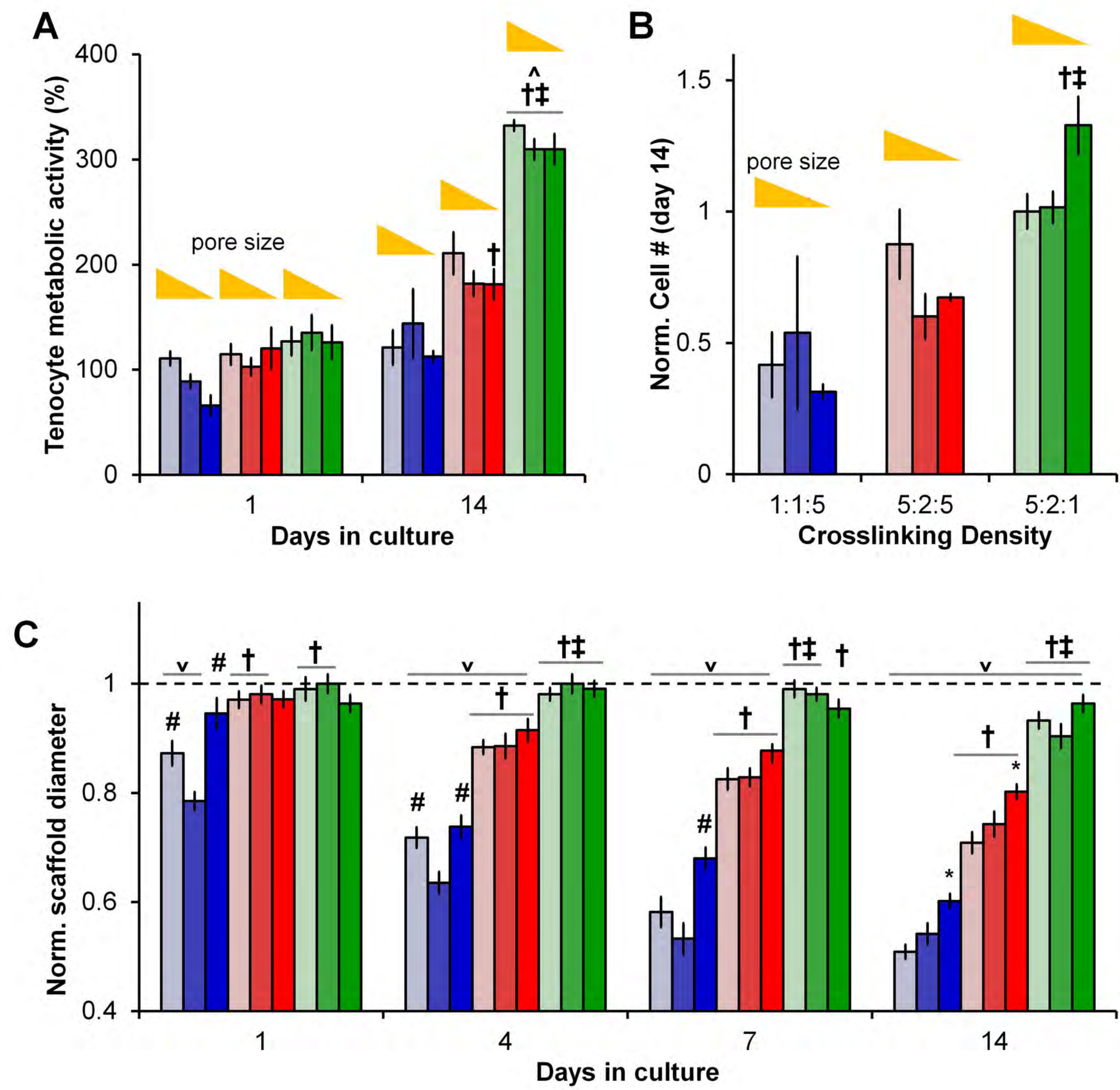
A

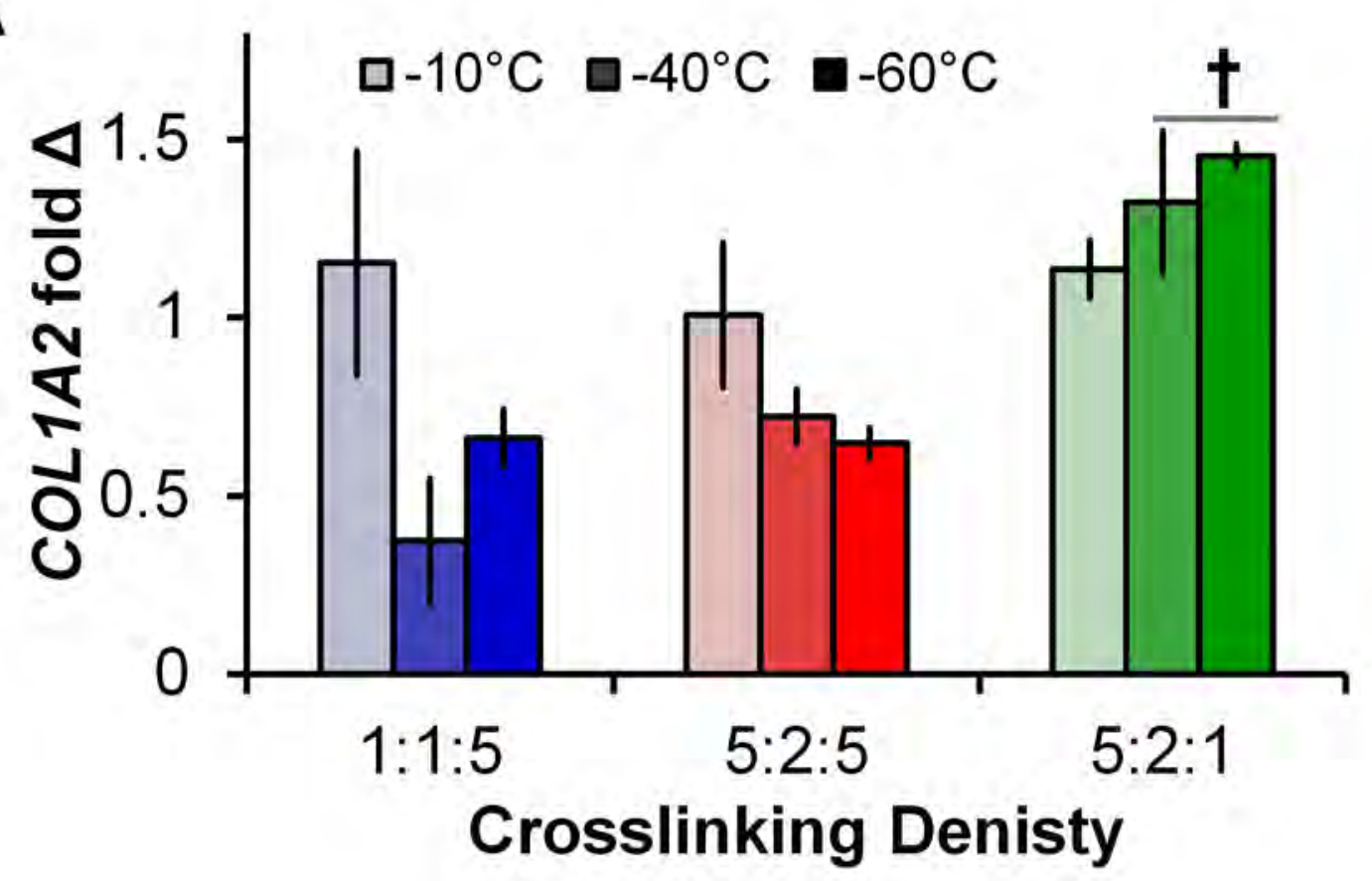

C

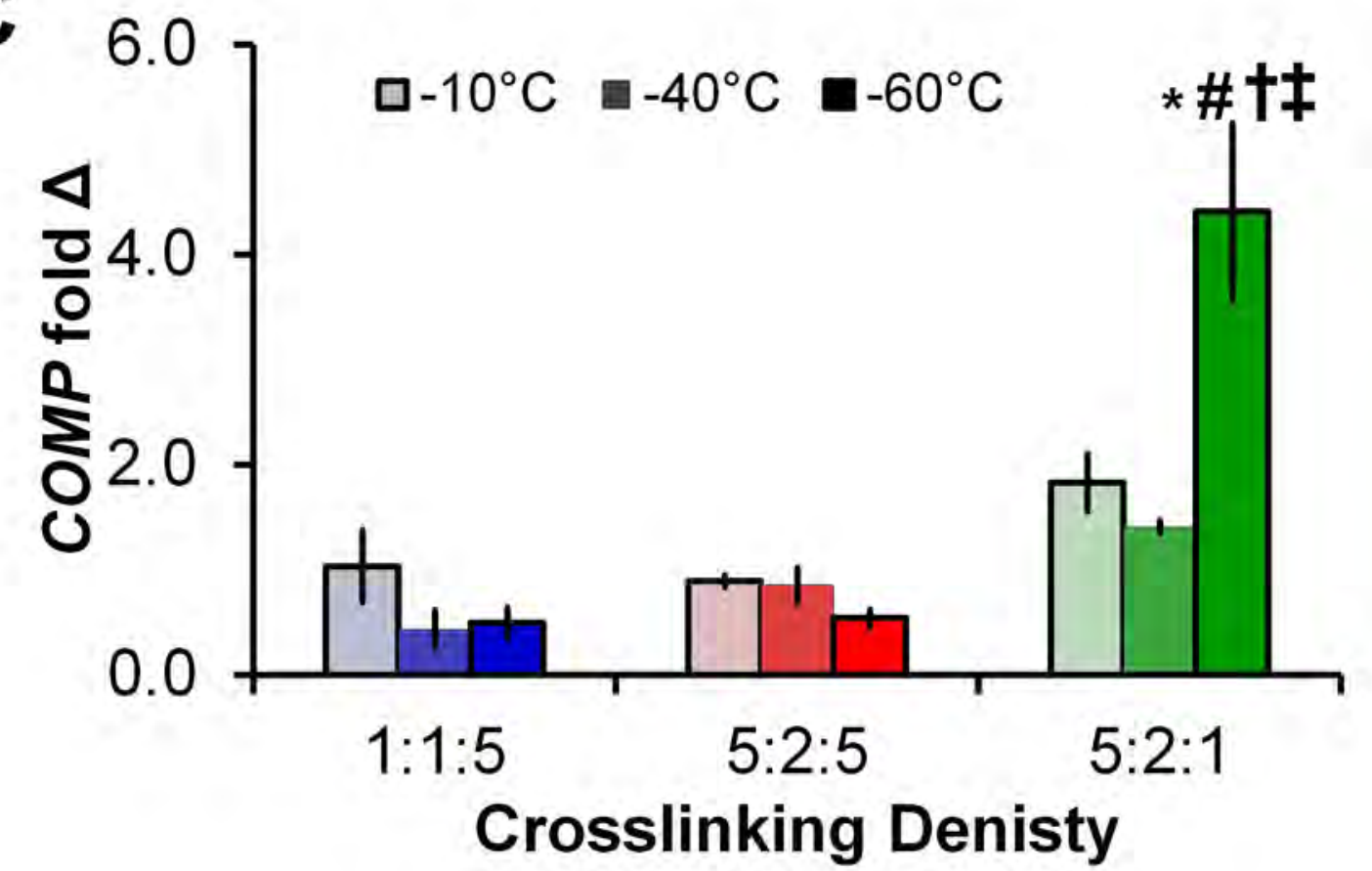

B
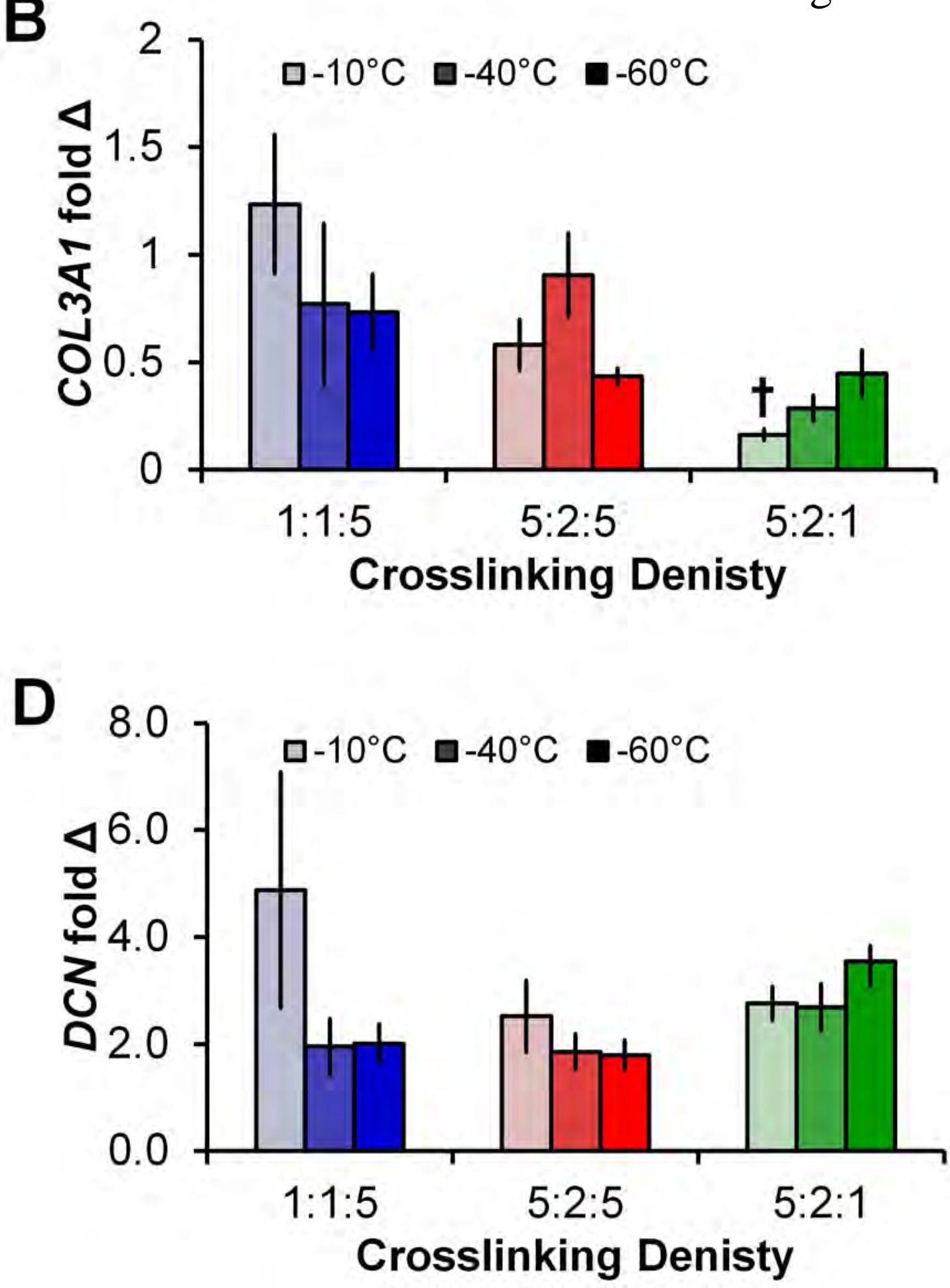
$\begin{array}{llll}\text { A } 100, & \text { B } & \text { Figure } 3\end{array}$
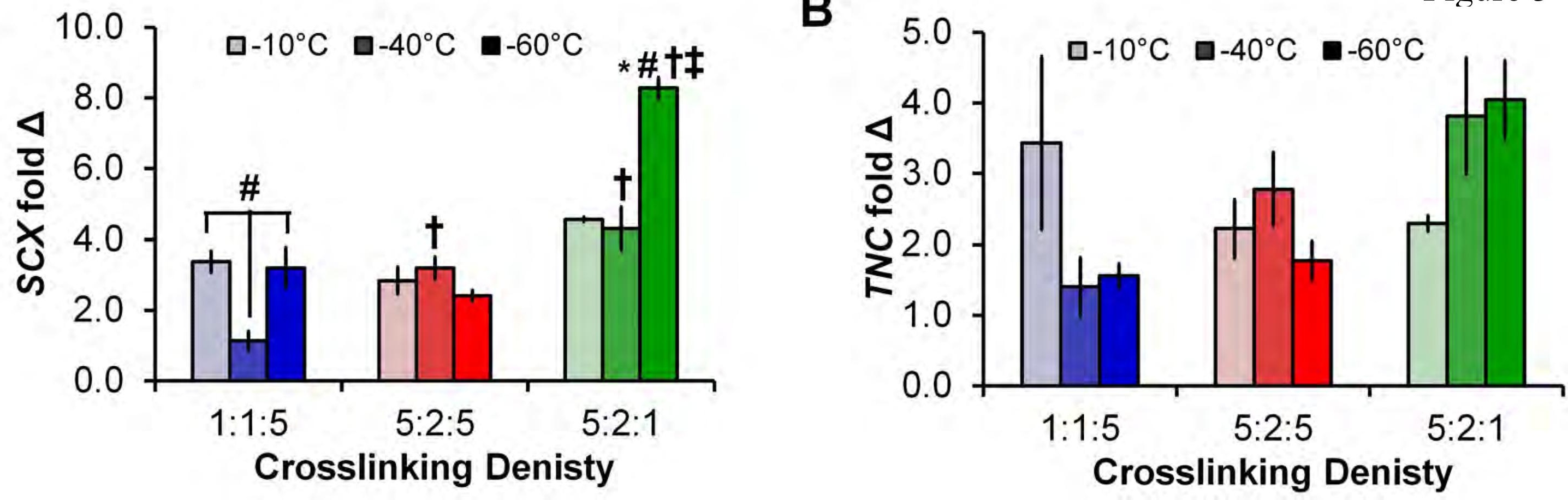
A $\quad$ Figure4

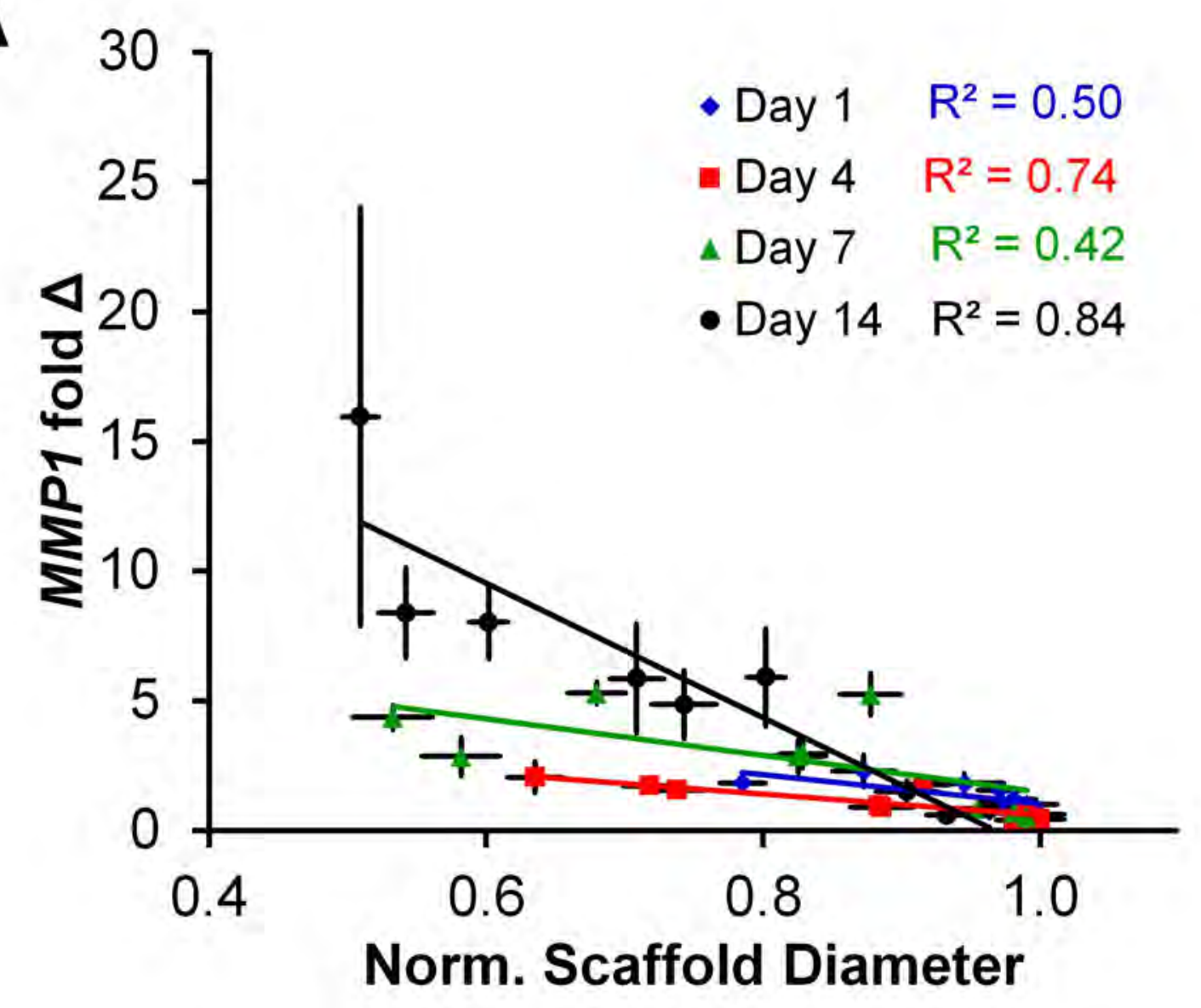

B

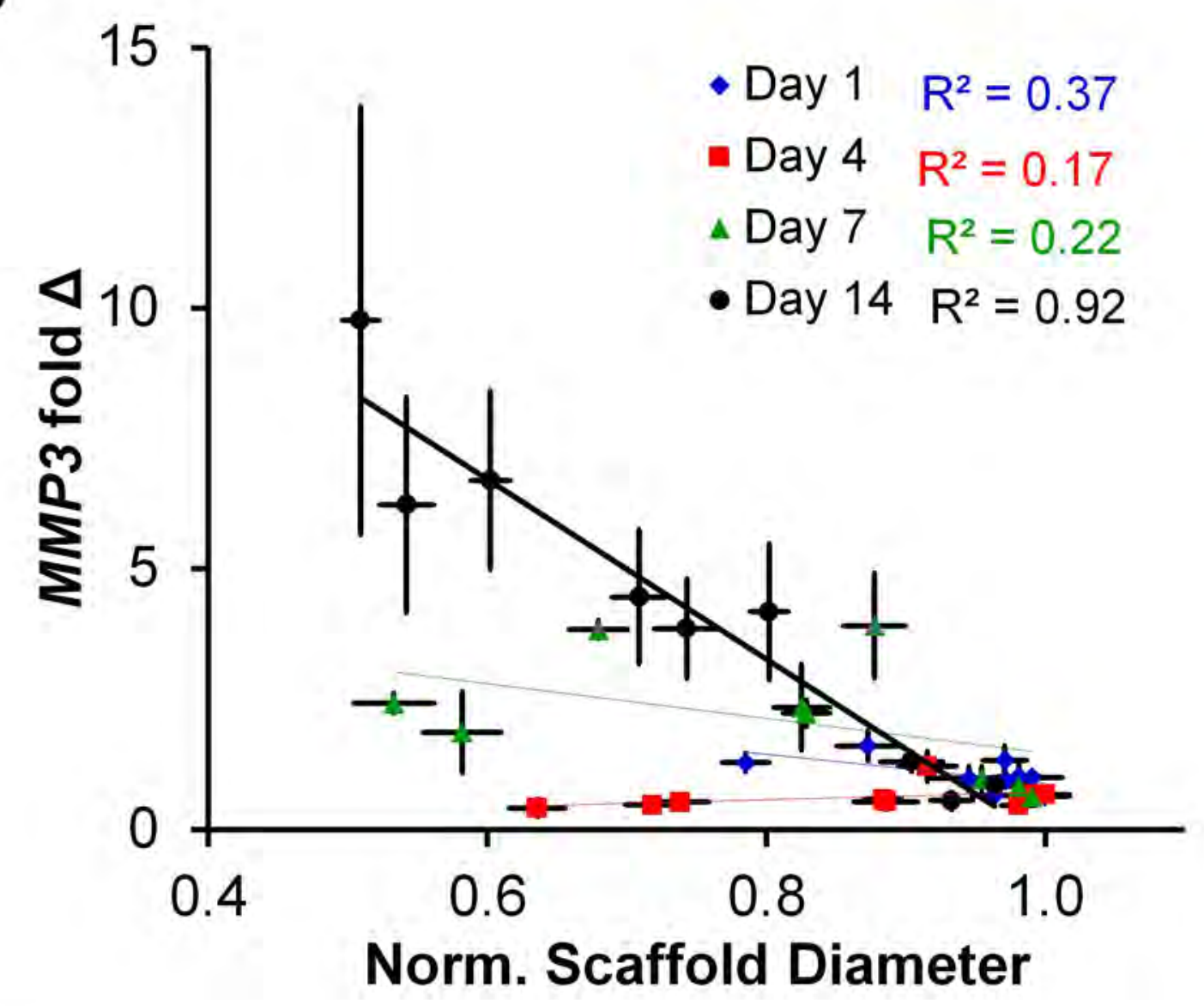

C

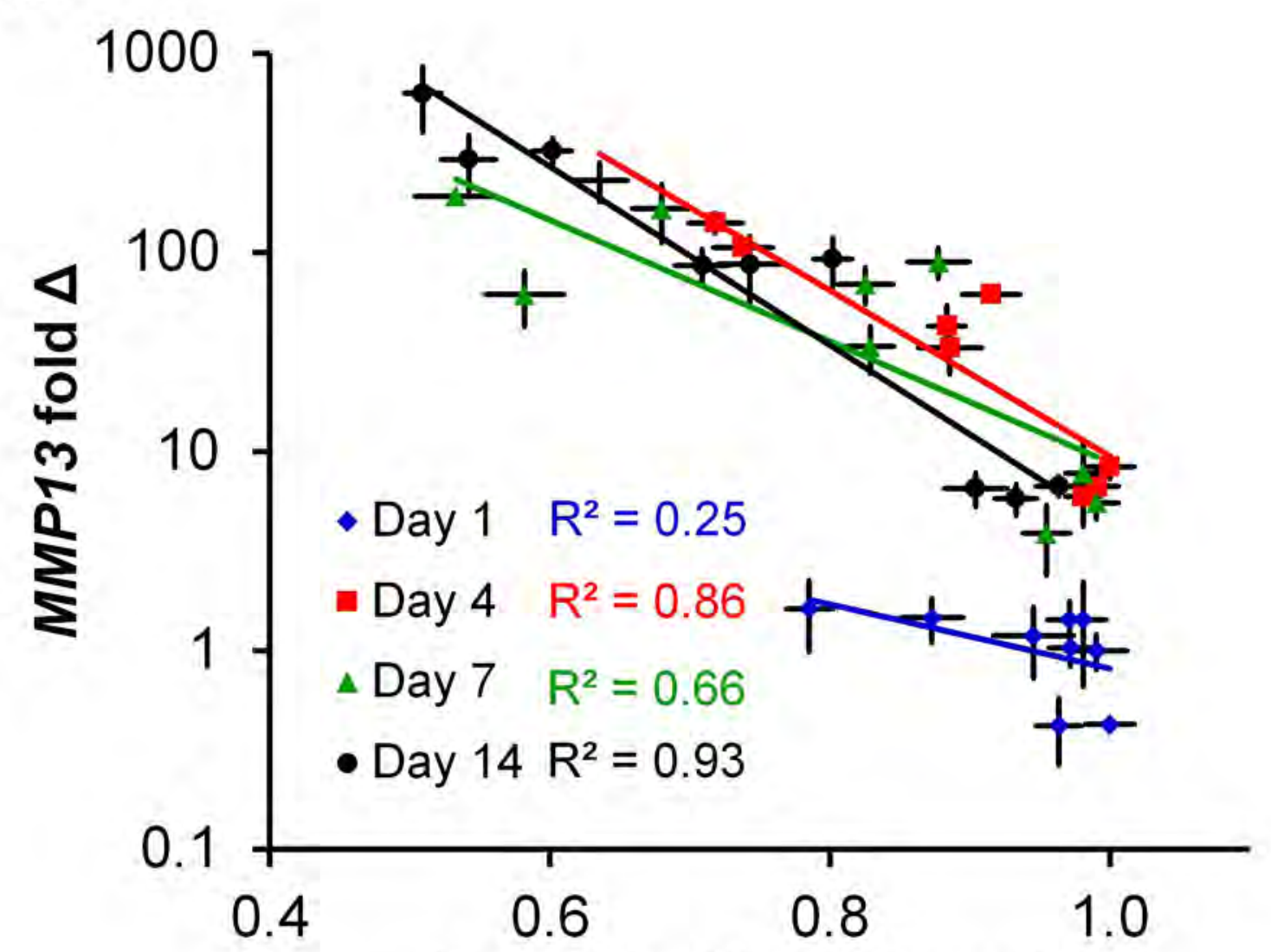

Norm. Scaffold Diameter 